\title{
Two Dimensional Electronic Nose for Vehicular Central Locking System (E-Nose-V)
}

\author{
Mahmoud Zaki Iskandarani \\ Faculty of Engineering, Al-Ahliyya Amman University, Amman, Jordan
}

\begin{abstract}
A new approach to vehicle security is proposed, tried, tested. The designed and tested system comprises an odor detection system (E-Nose) that sends signals corresponding to selected odors to the smart vehicle Electronic Control Unit (ECU), which is interfaced to a smart system with neural networks. The signal is interpreted in time and space, whereby a certain ordered number of samples should be obtained before the vehicle functions are unlocked. Correlation of rise and decay times and amplitudes of the signal is carried out to ensure security. The proposed system is highly secured and could be further developed to become a vital and integrated part of Intelligent Transportation Systems (ITS) through the addition of driver's body odor smell as an extra measure of security and in cases of accidents to auto call emergency services with driver identification and some diagnostics. Such system can be utilized in smart cities.
\end{abstract}

Keywords-Odors; E-Nose; neural networks; security; correlation; smart vehicles; intelligent transportation systems; smart cities

\section{INTRODUCTION}

An E-nose is generally recognized as a device that encompasses array of electrochemical sensors with specification capabilities and adaptable pattern recognition system, able to classify individual vapors components or combination of vapors. An electronic nose recognizes an odor through fingerprinting of its chemical elements using an array of sensors backed by intelligent software for pattern recognition. Such technology is already in use across many industries, including agricultural, environmental, food, manufacturing, and the military. Also, E-Noses are increasingly playing an important role in disease detection such as diabetes by means of blood sniffing and can detect lung diseases through sucking up vapors and sniffing the breath [15].

The E-Nose is a modular sensor system made for detection of odors, odor mixtures, aromas, and gaseous substances in both open and closed environments. It uses chemical sensors for detection and artificial intelligence for treatment of the measured data. Such a device is regarded as fingerprint one that analyze the odor as a whole. By processing the obtained fingerprint of the odor, that constituent substance(s) would be recognized and classified. Such functionality is important in many quality and security control systems [6-10].

There are two main components in the E-Nose; the sensing part and the intelligent pattern recognition part. The combination of broad sensing elements coupled with intelligent information processing algorithms makes the E-Nose such a promising tool for odor related applications. The sensing part comprises an array of chemical sensing elements with each sensing element capable of measuring a different part of the odor spectrum. Each odorant produces a unique characteristic (signature) of its own, once exposed to the sensing array. By presenting many different odorants to the sensor array, a database is built up. This database of odorants with each odorant unique characteristics is used to train an intelligent classification system, such as Neural Networks [11-20].

The way E-noses sense odorant molecules is similar to human olfactory system. The odors interact with a responsive materials making up the sensor array, resulting in a change in the material characteristics and producing a unique response related to the specific smelled odorant. The software processes the obtained signals, and filter out unnecessary information and performs pattern recognition. Some commonly used sensing materials are: metal oxides, conducting polymers and composites. Pattern recognition can be successfully performed using an Artificial Neural Networks (ANN) algorithm.

ANN is a learning and classification algorithm which is based on operation of the human brain. It deduces meaning from the data which is complex or difficult to mathematically represent in order to identify patterns and enable decision making. Weight Elimination Algorithm (WEA) is based on Backpropagation (BP) algorithm, which is an iterative gradient algorithm aims at decreasing the root mean square error. Input and output layers are interconnected through hidden layers.

Traditionally, systems are vulnerable to those armed with the right equipment and willing to intrude. Sometimes passwords are unchanged and left as the default ones. Access to these gives the power to the fraudster's to hack. The power of neural network- odor sensing based technology together with the traditional strengths of conventional rules-based fraud detection technologies is a crucial and very effective solution to intrusion, as adaptive neural analysis techniques allow high levels of security; while conventional rules-based technologies provide a high level of detail [21-30].

In this paper, a fresh approach to the use and application of E-Nose system is proposed which utilizes odor sensing together with artificial neural networks interfaced to the ECU of the vehicle. Such approach will support both driver and vehicle functionality in terms of driver identification for secure access and mobility. The system can be further developed to support driver health and status monitoring while driving and driver vanning, support and intervention under critical conditions if integrated with other vehicular systems and interfaced to wireless communication systems. 


\section{RELATED WORK}

The last few years seen a rapid development of Intelligent Transportation Systems (ITS), Intelligent Vehicles and Vehicular Ad Hoc Network (VANET) with the tendency to change the way we drive by providing a safe, secure, and reliable ubiquitous computing systems capable of covering many roads.

Due to the advancement in electronics and its interface and integrated into the automotive industry and modern transportation, there has been a marked change in road vehicle technology. In smart vehicles there are over 90 devices equivalent to microcomputers placed in each smart vehicle, which provide services to drivers by controlling vehicle operation, comfort, support, security and safety. Electronic devices are used control almost every function inside the vehicle and supervise complex and complicated.

An intelligent or smart vehicle, is a vehicle that is capable of sensing its environment by combining a variety of sensor inputs, such as RAdio Detection And Ranging (RADAR), LIDAR LIght Detection And Ranging (LIDAR), Global Positioning Systems (GPS),Computer vision devices such as Cameras, and other safety, mobility, comfort, and security related applications. Such applications are performed through continuous monitoring of the performance and status of the vehicle and driver. Such sensors can be classified under different categories such as; safety sensors, diagnostics sensors, multimedia and comfort sensors, environmental sensors, driver/driving monitoring sensors, and traffic monitoring sensors. There is no doubt that intelligent or smart vehicles will play a central role in personal mobility, driver safety and vehicle security in the future. Highly automated driving systems will relieve the drivers from stressing tasks ensuring safer and sustainable mobility [31].

Three main operational levels of sensor based electronic computing systems are realized within an intelligent vehicle system [32]:

1) Warning: To inform the driver about potentially dangerous situations without support or intervention.

2) Support: To inform and support the driver and provide guidance in facing a risky and threatening situation.

3) Intervention: An automated intervention into the vehicle control is carried out at this level.

In intelligent vehicles, the objective is to fuse and aggregate data from different sensors according to the following criteria:

a) Data source.

b) Data type.

c) Data architecture.

d) Data level of abstraction.

Data type and sources are critical in facing many challenges related to transportation systems, such as smooth integration independent data sources in real-time to meet requirements.

Data integration techniques must be integrated with Artificial Intelligence algorithms (AI) to allow the vehicle to understand the current state similar to human understanding and interpretation, and to react accordingly.

The privacy and security of vehicles highly depend on reducing intrusiveness by including security and privacy protocols into the communication devices that are supported by the communication networks to protect drivers, vehicles, passengers, and passengers within the vehicular network environment. Hackers have penetrated, modified, corrupted or taken control of vehicle systems through sensors monitoring systems and internal and external vehicle communication systems. Threats exist to identity data in vehicles with intrusion with malicious intent. In-vehicle software can have millions of lines of code which executes on both the primary computer board(s) and over 80 microprocessor-based electronic control units (ECUs) networked throughout the body of the car [3343].

It is envisaged in this work that it could be very useful to produce mobile E-Noses that could also be mounted within a vehicular structure (On Board Units OBU), which is interfaced to the rest of the vehicle via and ECU unit and can work in collaboration with the rest of the vehicle sensory system. An Enose device attached to an intelligent car is considered a sensory source similar to on board cameras, radar and other sensor data sources, which all need specialized software for data collection and interpretation. Adding E-noses to the system of available sensors will lead to improvement of the overall security model on the three mentioned operational levels of the vehicle computing system aiding in both driver identification, health diagnostics such as stress levels, heart attacks, accidents, and vehicle security.

\section{MATERIALS AND MethodS}

Three Odor keys are inserted and form a complex odor key by combining the sequential sampling of the three separate odor keys into one correlated odor key. The odor detection system employs Figaro TGS (Metal Oxide Semiconductor) Sensors shown in Fig. 1. The keys inserted in a certain order as an extra measure of security. The sampling process is shown in Fig. 2. The system will sample the odor keys until it returns back to its original base signal after completing three-key scan, before unlocking.

Weight Elimination Algorithm (WEA) is used to carry out training of the Neural Networks system in order to enable unlocking and locking of the vehicle as shown in Fig. 3.

WEA works by minimizing a modified error function which is formed by adding an overhead term to the original error function used in the algorithm. The overhead variable in weight decay applies cost values to large weights, hence, causes weights under consideration to converge to smaller absolute values. Large weights can adversely affect generalization according to their position in the network. When large weight values lie between input layer and hidden layer, they affect smoothness of the output function, with possible near discontinuities. However, if they lie between the hidden layer and the output layer, they can lead to out of range outputs. Hence, large weights can cause excessive variation in the output and instability of the neural structure, as their values and instability will be outside the range of the output activation 
function. Weights size is critical and in some cases will have marked effect in determining generalization.

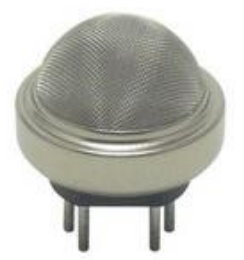

Fig. 1. Figrao TGS (MOS) Sensor.

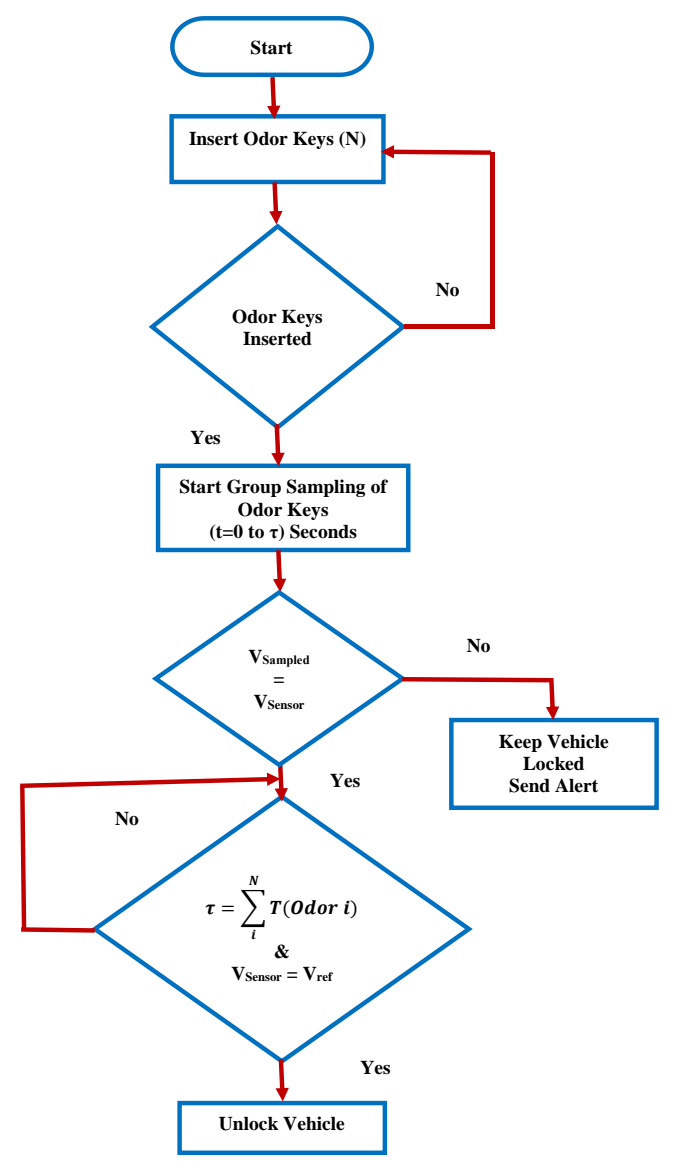

Fig. 2. Odor Key Unlocking System

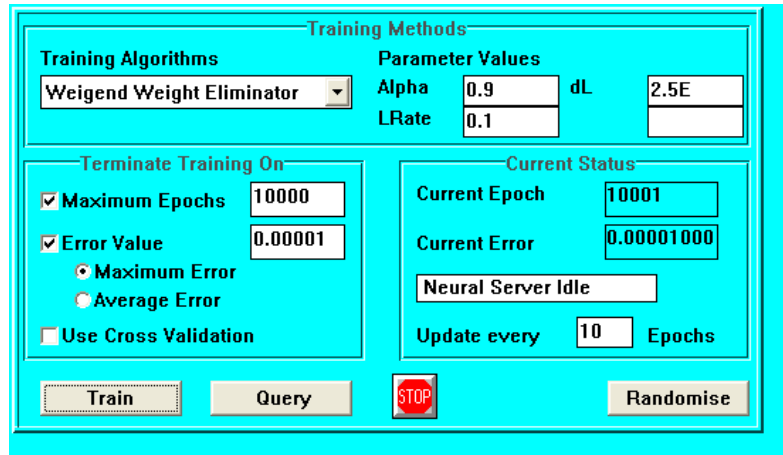

Fig. 3. Neural Networks (WEA) Traninig System
Weight elimination describes the dynamic changes in neural network convergence through error functions. The overall weight elimination error function is shown in equation (1):

$E_{W E}=E_{\text {Initial }}+E_{\text {Overhead }}$

Where;

$E_{\text {initial }}=\frac{1}{2} \sum_{k}\left(d_{k}-o_{k}\right)^{2}$

$E_{\text {Overhead }}=\beta\left(\sum_{j k} \frac{\left(\frac{w_{j k}}{w_{N}}\right)^{2}}{1+\left(\frac{w_{j k}}{w_{N}}\right)^{2}}\right)$.

Where;

$E_{W E}$ : The combined overhead function that includes the initial overhead function, $E_{\text {Initial }}$ and the weight-elimination term $E_{\text {Overhead }}$.

$\beta$ : The weight-reduction factor,

$w_{j k}$ : Represents the individual weights of the neural network model

$\mathcal{W}_{N}$ : A scale parameter computed by the WEA.

$d_{k}:$ The desired Output.

$O_{k}:$ The actual Output.

The dynamic weight changes is calculated as shown in equation (4)

$\Delta w_{j k}=\left(-\alpha \frac{\partial E_{\text {Initial }}}{\partial w_{j k}}\right)-\left(\beta \frac{\partial E_{\text {Overhead }}}{\partial w_{j k}}\right)$

Where;

$\alpha$ : The Learning Rate (between 0 and 1)

The parameter, $w_{N}$, is a scale parameter computed by the WEA, and selected to be the smallest weight from the last epoch or set of epochs to converge small weights to zero. $w_{N}$ plays an fundamental role so that the network will converge with either small number of large weights or large number of small weights. 


\section{RESUlts}

Tables I to III show the used odor keys used in validating the designed system, while Fig. 4 shows the Neural Network model used for training with distributed weights.

TABLE I. USED ODOR KEY 1

\begin{tabular}{|l|l|}
\hline Odor Key 1 & \\
\hline Response Time (Seconds) & VSensor/VRef \\
\hline 0 & 1 \\
\hline 1 & 1.3 \\
\hline 2 & 1 \\
\hline
\end{tabular}

TABLE II. USED ODOR KEY 2

\begin{tabular}{|l|l|}
\hline Odor Key 2 & \\
\hline Response Time (Seconds) & VSensor/VRef \\
\hline 0 & 1 \\
\hline 1 & 1.4 \\
\hline 2 & 2.6 \\
\hline 3 & 2.7 \\
\hline 4 & 2.55 \\
\hline 5 & 1.9 \\
\hline 6 & 1.6 \\
\hline 7 & 1.4 \\
\hline 8 & 1.25 \\
\hline 9 & 1 \\
\hline
\end{tabular}

TABLE III. USED ODOR KEY 3

\begin{tabular}{|c|c|}
\hline \multicolumn{2}{|l|}{ Odor Key 3} \\
\hline Response Time (Seconds) & VSensor/VRef \\
\hline 0 & 1 \\
\hline 1 & 1.3 \\
\hline 2 & 2 \\
\hline 3 & 3.2 \\
\hline 4 & 3.7 \\
\hline 5 & 3.7 \\
\hline 6 & 3.7 \\
\hline 7 & 3.7 \\
\hline 8 & 3.7 \\
\hline 9 & 2.7 \\
\hline 10 & 2 \\
\hline 11 & 1.8 \\
\hline 12 & 1.6 \\
\hline 13 & 1.5 \\
\hline 14 & 1.4 \\
\hline 15 & 1.25 \\
\hline 16 & 1.1 \\
\hline 17 & 1.05 \\
\hline 18 & 1 \\
\hline
\end{tabular}

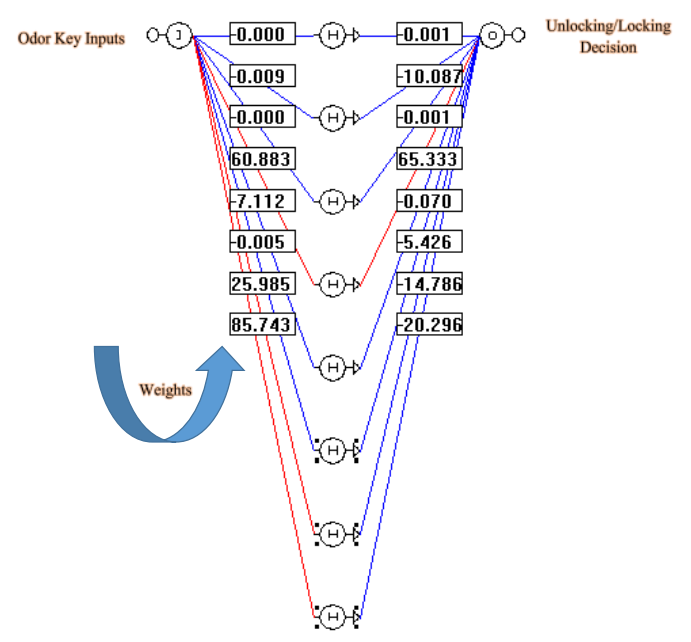

Fig. 4. Neural Networks Model used for Traning and Decision Making.

\section{ANALYSIS AND DISCUSSION}

Fig. 5 shows the signal characteristics of odor coded key, which consists of three separate odor keys combined.

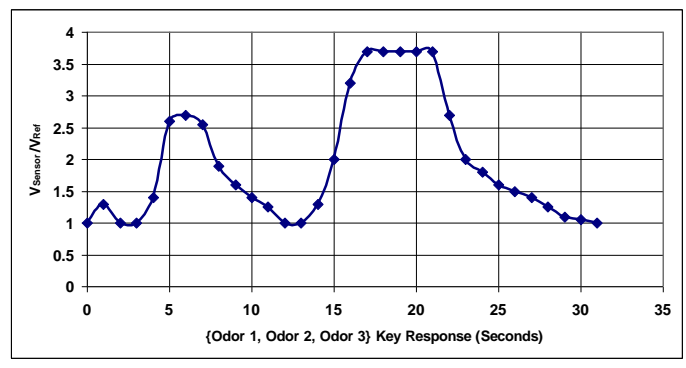

Fig. 5. Used Odor Coded Key in System Testing.

Table IV shows used training Odor keys data for the Neural Networks model shown in Fig. 4, while Table V shows the testing results of the Neural System assuming the correct Odor Keys are used.

Tables VI and VII shows testing data obtained as a result of incorrect odor keys 2 and 3 with Table VIII and Fig. 6 showing areas of pattern change and difference between correct and incorrect odor keys.

As the sampled odor keys that form the complex odor key change in pattern, the inputs to the neural network will change, causing a different actual output which when compared with desired output will result in a unique value that causes the system to stop sampling and raise an alarm and send an alert to the vehicle owner that an unauthorized key is being used. The vehicle system will stay locked and a time stamp will register the date and time the incident occurred.

Under normal operating conditions, the sampling system should fulfill both time cycle scanning, amplitude cycle scanning, and order cycle scanning to allow unlocking of the vehicle and its functions, hence, the result is a three dimensional function as prescribed by equation (5).

$$
f_{\text {unlocking }}=f(\text { time, amplitude, position }) \text {. }
$$


TABLE IV. OdOR Keys TRAINING DATA

\begin{tabular}{|c|c|c|}
\hline \multicolumn{3}{|c|}{ Training Data Set $\{$ Odor 1 , Odor 2 , Odor 3$\}$} \\
\hline $\begin{array}{l}\text { Response Time } \\
\text { (Seconds) }\end{array}$ & $\begin{array}{l}\text { Training Data } \\
\text { Input } \\
\text { VSensor/VRef }\end{array}$ & $\begin{array}{l}\text { Training Data } \\
\text { Output } \\
\text { VSensor/VRef }\end{array}$ \\
\hline 0 & 1 & 0 \\
\hline 1 & 1.3 & 1 \\
\hline 2 & 1 & 0 \\
\hline 3 & 1 & 0 \\
\hline 4 & 1.4 & 1 \\
\hline 5 & 2.6 & 1 \\
\hline 6 & 2.7 & 1 \\
\hline 7 & 2.55 & 1 \\
\hline 8 & 1.9 & 1 \\
\hline 9 & 1.6 & 1 \\
\hline 10 & 1.4 & 1 \\
\hline 11 & 1.25 & 1 \\
\hline 12 & 1 & 0 \\
\hline 13 & 1 & 0 \\
\hline 14 & 1.3 & 1 \\
\hline 15 & 2 & 1 \\
\hline 16 & 3.2 & 1 \\
\hline 17 & 3.7 & 1 \\
\hline 18 & 3.7 & 1 \\
\hline 19 & 3.7 & 1 \\
\hline 20 & 3.7 & 1 \\
\hline 21 & 3.7 & 1 \\
\hline 22 & 2.7 & 1 \\
\hline 23 & 2 & 1 \\
\hline 24 & 1.8 & 1 \\
\hline 25 & 1.6 & 1 \\
\hline 26 & 1.5 & 1 \\
\hline 27 & 1.4 & 1 \\
\hline 28 & 1.25 & 1 \\
\hline 29 & 1.1 & 1 \\
\hline 30 & 1.05 & 1 \\
\hline 31 & 1 & 0 \\
\hline
\end{tabular}

TABLE V. Odor Keys Testing Data: CORRECT Keys

\begin{tabular}{|c|c|c|c|}
\hline \multicolumn{4}{|c|}{ Testing Data Set 1 \{Odor 1 , Odor 2, Odor 3$\}$} \\
\hline $\begin{array}{l}\text { Response Time } \\
\text { (Seconds) }\end{array}$ & $\begin{array}{l}\text { Testing Data } \\
\text { Input } \\
\text { VSensor/VRef }\end{array}$ & $\begin{array}{l}\text { Testing Data } \\
\text { Output } \\
\text { VSensor/VRef }\end{array}$ & $\begin{array}{l}\text { Rounded } \\
\text { Output }\end{array}$ \\
\hline 0 & 1 & 0 & 0 \\
\hline 1 & 1.3 & 1.00197 & 1 \\
\hline 2 & 1 & 0.00006 & 0 \\
\hline 3 & 1 & 0.00006 & 0 \\
\hline 4 & 1.4 & 1.00197 & 1 \\
\hline 5 & 2.6 & 0.99917 & 1 \\
\hline 6 & 2.7 & 0.99959 & 1 \\
\hline 7 & 2.55 & 0.99943 & 1 \\
\hline 8 & 1.9 & 0.99987 & 1 \\
\hline 9 & 1.6 & 0.99917 & 1 \\
\hline 10 & 1.4 & 1.00197 & 1 \\
\hline 11 & 1.25 & 0.99788 & 1 \\
\hline 12 & 1 & 0.00006 & 0 \\
\hline 13 & 1 & 0.00006 & 0 \\
\hline 14 & 1.3 & 1.00197 & 1 \\
\hline 15 & 2 & 0.99877 & 1 \\
\hline 16 & 3.2 & 1.00003 & 1 \\
\hline 17 & 3.7 & 1.00045 & 1 \\
\hline 18 & 3.7 & 1.00045 & 1 \\
\hline 19 & 3.7 & 1.00045 & 1 \\
\hline 20 & 3.7 & 1.00045 & 1 \\
\hline 21 & 3.7 & 1.00045 & 1 \\
\hline 22 & 2.7 & 0.99959 & 1 \\
\hline 23 & 2 & 0.99877 & 1 \\
\hline 24 & 1.8 & 0.99862 & 1 \\
\hline 25 & 1.6 & 0.99917 & 1 \\
\hline 26 & 1.5 & 1.00021 & 1 \\
\hline 27 & 1.4 & 1.00197 & 1 \\
\hline 28 & 1.25 & 0.99788 & 1 \\
\hline 29 & 1.1 & 1.00180 & 1 \\
\hline 30 & 1.05 & 0.99867 & 1 \\
\hline 31 & 1 & 0.00006 & 0 \\
\hline
\end{tabular}


TABLE VI. OdOR KeYS TESTING DATA-CHANGE IN ODOR KEY 2

\begin{tabular}{|l|l|l|l|}
\hline $\begin{array}{l}\text { Testing Data Set } 2 \\
\text { Incorrect Odor Key } 3\end{array}$ Odor 1, Odor 2, Odor 3\} \\
\hline $\begin{array}{l}\text { Response Time } \\
\text { (Seconds) }\end{array}$ & $\begin{array}{l}\text { Testing Data } \\
\text { Input } \\
\text { VSensor/VRef }\end{array}$ & $\begin{array}{l}\text { Actual Testing } \\
\text { Data Output } \\
\text { VSensor/VRef }\end{array}$ & $\begin{array}{l}\text { Rounded } \\
\text { Output }\end{array}$ \\
\hline 0 & 1 & 0 & 0 \\
\hline 1 & 1.3 & 1.00197 & 1 \\
\hline 2 & 1 & 0.00006 & 0 \\
\hline 3 & 1 & 0.00006 & 0 \\
\hline 4 & 1.4 & 1.00197 & 1 \\
\hline 5 & 0.6 & -0.09999 & 0 \\
\hline Time Stamp & \multicolumn{2}{l}{} \\
\hline
\end{tabular}

TABLE VII. Odor Keys Testing DATA-Change IN Odor Key 3

\begin{tabular}{|c|c|c|c|}
\hline \multicolumn{4}{|c|}{$\begin{array}{l}\text { Testing Data Set } 3 \text { \{Odor 1, Odor 2, Odor } 3\} \\
\text { Incorrect Odor Key } 3\end{array}$} \\
\hline $\begin{array}{l}\text { Response Time } \\
\text { (Seconds) }\end{array}$ & $\begin{array}{l}\text { Testing Data } \\
\text { Input } \\
\text { VSensor/VRef }\end{array}$ & $\begin{array}{l}\text { Actual Testing } \\
\text { Data Output } \\
\text { VSensor/VRef }\end{array}$ & $\begin{array}{l}\text { Rounded } \\
\text { Output }\end{array}$ \\
\hline 0 & 1 & 0 & 0 \\
\hline 1 & 1.3 & 1.00197 & 1 \\
\hline 2 & 1 & 0.00006 & 0 \\
\hline 3 & 1 & 0.00006 & 0 \\
\hline 4 & 1.4 & 1.00197 & 1 \\
\hline 5 & 2.6 & 0.99917 & 1 \\
\hline 6 & 2.7 & 0.99959 & 1 \\
\hline 7 & 2.55 & 0.99943 & 1 \\
\hline 8 & 1.9 & 0.99987 & 1 \\
\hline 9 & 1.6 & 0.99917 & 1 \\
\hline 10 & 1.4 & 1.00197 & 1 \\
\hline 11 & 1.25 & 0.99788 & 1 \\
\hline 12 & 1 & 0.00006 & 0 \\
\hline 13 & 1 & 0.00006 & 0 \\
\hline 14 & 1.3 & 1.00197 & 1 \\
\hline 15 & 2 & 0.99877 & 1 \\
\hline 16 & 3.2 & 1.00003 & 1 \\
\hline 17 & 3.7 & 1.00045 & 1 \\
\hline 18 & 3.7 & 1.00045 & 1 \\
\hline 19 & 3.7 & 1.00045 & 1 \\
\hline 20 & 3.7 & 1.00045 & 1 \\
\hline 21 & 3.7 & 1.00045 & 1 \\
\hline 22 & 2.7 & 0.99959 & 1 \\
\hline 23 & 2 & 0.99877 & 1 \\
\hline 24 & 1.8 & 0.99862 & 1 \\
\hline 25 & 1.6 & 0.99917 & 1 \\
\hline 26 & 0.5 & -0.09999 & 0 \\
\hline Time Stamp & Stop Sampling & end Alert & \\
\hline
\end{tabular}

TABLE VIII. COMPARISON BETWEEN OdOR KeYS TESTING DATA

\begin{tabular}{|c|c|c|c|}
\hline \multicolumn{4}{|c|}{ All Data Sets \{Odor 1, Odor 2, Odor 3$\}$} \\
\hline $\begin{array}{l}\text { Response Time } \\
\text { (Seconds) }\end{array}$ & $\begin{array}{l}\text { Correct } \\
\text { Odor Keys } \\
\text { VSensor/VRef }\end{array}$ & $\begin{array}{l}\text { Incorrect } \\
\text { Odor Key } 2 \\
\text { VSensor/VRef }\end{array}$ & $\begin{array}{l}\text { Incorrect } \\
\text { Odor Key } 3 \\
\text { VSensor/VRef }\end{array}$ \\
\hline 0 & 1 & 1 & 1 \\
\hline 1 & 1.3 & 1.3 & 1.3 \\
\hline 2 & 1 & 1 & 1 \\
\hline 3 & 1 & 1 & 1 \\
\hline 4 & 1.4 & 1.4 & 1.4 \\
\hline 5 & 2.6 & 0.6 & 2.6 \\
\hline 6 & 2.7 & & 2.7 \\
\hline 7 & 2.55 & & 2.55 \\
\hline 8 & 1.9 & & 1.9 \\
\hline 9 & 1.6 & & 1.6 \\
\hline 10 & 1.4 & & 1.4 \\
\hline 11 & 1.25 & & 1.25 \\
\hline 12 & 1 & & 1 \\
\hline 13 & 1 & & 1 \\
\hline 14 & 1.3 & & 1.3 \\
\hline 15 & 2 & & 2 \\
\hline 16 & 3.2 & & 3.2 \\
\hline 17 & 3.7 & & 3.7 \\
\hline 18 & 3.7 & & 3.7 \\
\hline 19 & 3.7 & & 3.7 \\
\hline 20 & 3.7 & & 3.7 \\
\hline 21 & 3.7 & & 3.7 \\
\hline 22 & 2.7 & & 2.7 \\
\hline 23 & 2 & & 2 \\
\hline 24 & 1.8 & & 1.8 \\
\hline 25 & 1.6 & & 1.6 \\
\hline 26 & 1.5 & & 0.5 \\
\hline 27 & 1.4 & & \\
\hline 28 & 1.25 & & \\
\hline 29 & 1.1 & & \\
\hline 30 & 1.05 & & \\
\hline 31 & 1 & & \\
\hline
\end{tabular}

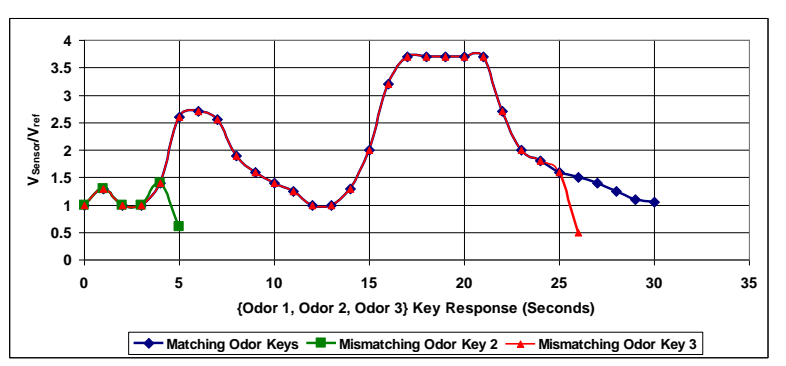

Fig. 6. System Testing with Incorrect Odor Keys. 
Any deviation in any of the parameters in equation (5) will cause the system to halt and alert. The order or keys position relative to each other can periodically be change, by retraining the system again for different order of the same odorant, which is a standard security practice as changing a password every few weeks. Also, the types of odorants can also be changed and the system retrained to use the new odorants, which is a fourth security dimension.

The sampling system could be placed in a remote device held by the person concerned or in the vehicle itself with regular but random interchanging of odor keys.

Neural Engine will initially be trained to recognize the odor keys and only unlocks the vehicle if the random combination of the three keys falls within the space-time domains of the training key sets. Any other keys will not be able to unlock the system and will raise a remote call to the user.

\section{CONCLUSIONS}

The proposed system is a good step towards a new type of vehicular security systems, which can be made as simple and as complex as necessary by increasing the number of odorants or the process of sampling. Future developments could include smelling of the driver body odorant in a combination with an external odorant or on its own, which means that body odor can be used as a biometric identification for vehicles, similar to fingerprinting. More stable and integrated sensors with temperature compensation and high level of noise isolation will contribute to a great deal in refining the system and improve its reliability.

\section{REFERENCES}

[1] J. Yan, X. Guo, S. Duan, P. Jia, L. Wang, C. Peng, S. Zhang, "Electronic nose feature extraction methods: A review," Sensors, vol. 15, pp. 27804-27831, 2015.

[2] S. Vishesh, M. Srinath, K. Gubbi, H. Shivu, N. Prashanta, "Portable Low Cost Electronic Nose for Instant and Wireless Monitoring of Emission Levels of Vehicles Using Android Mobile Application," IJARCCE, vol. 5, no. 9, pp. 134-140, 2016.

[3] R. Suganya, R. Uthayakumar"Electronic nose for accident prevention and vehicleblack box system," IJARECE, vol. 4, no. 5, pp. 1206-1209, 2015.

[4] A. Guentner, V. Koren, K. Chikkadi, M. Righettoni, S. Pratsinis, "Enose sensing of low-ppb formaldehyde in gas mixtures at high relative humidity for breath screening of lung cancer?" ACS Sensors, vol. 1, no. 5, pp. 528-535, 2016.

[5] A. Loutfi, S. Coradeschi, G. Mani, P. Shanker, J. Rayappan, "Electronic noses for food quality: a review," Journal of Food Engineering, vol. 144, pp. 103-111, 2015.

[6] A. Gongora, J. Monroy, J. Gonzalez-Jimenez, "An Electronic Architecture for Multipurpose Artificial Noses," Journal of Sensors, vol. 2018, pp. 1-9, 2018.

[7] H. Zhiyi, H. Chenchao, Z. Jiajia, H. Guohua, "Electronic nose system fabrication and application in large yellow croaker (Pseudosciaena crocea) fressness prediction," Journal of Food Measurement and Characterization, vol. 11, no. 1, pp. 33-40, 2017.

[8] M. Castrica, S. Panseri, E. Siletti, F. Borgonovo, L. Chiesa, C. Balzaretti, "Evaluation of Smart Portable Device for Food Diagnostics: A Preliminary Study on Cape Hake Fillets (M. capensis and M. paradoxus)," Journal of Chemistry, vol. 2019, Article ID 2904724, pp. $1-7,2019$.
[9] S. Chiu, K. Tang, "Towards a chemiresistive sensorintegrated electronic nose: a review," Sensors, vol. 13, no. 10, pp. 14214-14247, 2013.

[10] J. Guti'errez, M. Horrillo, "Advances in artificial olfaction: Sensors and applications," Talanta, vol. 124, pp. 95-105, 2014.

[11] S. Marco, A. Guti'errez-G'alvez, A. Lansner et al, "A biomimetic approach to machine olfaction, featuring a very large-scale chemical sensor array and embedded neuro-bio-inspired computation," Microsystem Technologies, vol. 20, no. 4-5, pp. 729-742, 2014.

[12] L. Xu, J. He, S. Duan, X. Wu, Q. Wang, "Comparison of machine learning algorithms for concentration detection and prediction of formaldehyde based on electronic nose," Sensor Review, vol. 36, no.2, pp. 207-216, 2016.

[13] S. Radi, S. Ciptohadijoyo, W. Litananda, M. Rivai, M. Purnomo, "Electronic nose based on partition column integrated with gas sensor for fruit identification and classification," Computers and Electronics in Agriculture, vol. 121, pp. 429-435, 2016.

[14] S. Sarkar, A. Bhondekar, M. Macas et al., "Towards biological plausibility of electronic noses:A spiking neural network based approach for tea odour classification," Neural Networks, vol. 71, pp. 142-149, 2015.

[15] Y. Sun, D. Luo, H. Li, C. Zhu, O. Xu, H. Hosseini, "Detecting and Identifying Industrial Gases by a Method Based on Olfactory Machine at Different Concentrations," Journal of Electrical and Computer Engineering, vol. 2018, Article ID 1092718, pp. 1-9, 2018.

[16] E. Ordukaya, B. Karlik, "Quality Control of Olive Oils Using Machinem Learning and Electronic Nose," Journal of Food Quality, vol. 2017, Article ID 9272404, pp. 1-7, 2017.

[17] A. Tiele, S. Esfahani, J. Covington, "Design and Development of a Low-Cost, Portable Monitoring Device for Indoor Environment Quality," Journal of Sensors, vol. 2018, Article ID 5353816, pp. 1-14, 2018.

[18] A. Iosifidis, A. Tefas, and I. Pitas, "Approximate kernel extreme learning machine for large scale data classification," Neurocomputing, vol. 219, pp. 210-220, 2017.

[19] K. Yan, D. Zhang, "Calibration transfer and drift compensation of enoses via coupled task learning," Sensors and Actuators B: Chemical, vol. 225, pp. 288-297, 2016.

[20] Z. Ma, G. Luo, K. Qin, N. Wang, W. Niu, "Weighted Domain Transfer Extreme Learning Machine and Its Online Version for Gas Sensor Drift Compensation in E-Nose Systems," Wireless Communications and Mobile Computing, vol. 2018, Article ID 2308237, pp. 1-17, 2018.

[21] A. Di Gilio, J. Palmisani, G. de Gennaro," An Innovative Methodological Approach for Monitoring and Chemical Characterization of Odors around Industrial Sites," Advances in Meteorology, vol. 2018, Article ID 1567146, pp. 1-8, 2018.

[22] A. Hassan, A. Bermak, "Biologically Inspired Feature Rank Codes for Hardware Friendly Gas Identification with the Array of Gas Sensors," IEEE Sens. J, vol. 16, pp. 5776-5784, 2016.

[23] Y. Jing, Q. Meng, P. Qi, M. Cao, Z. Ming, S. Ma, "A Bioinspired Neural Network or Data Processing in an Electronic Nose," IEEE Trans. Instrum. Meas., vol. 65, pp. 2369-2380, 2016.

[24] L. Zhang, F. Tian, G. Pei, "A novel sensor selection using pattern recognition in electronic nose," Measurement, vol. 54, pp. 31-39, 2014.

[25] H. Ray, N. Bhattacharyya, A. Ghosh, B. Tudu, R. Bandyopadhyay, S. Biswas, S. Majumdar, "Fragrance profiling of Jasminum sambac Ait. flowers using electronic nose," IEEE Sens. J, vol. 17, pp. 160-168, 2017.

[26] H. Liu, Q. Li, B. Yan, L. Zhang, Y. Gu, "Bionic Electronic Nose Based on MOS Sensors Array and Machine Learning Algorithms Used for Wine Properties Detection," Sensors, vol. 19, no. 45, pp. 1-11, 2019.

[27] W. Cao, C. Liu, S. Duan, P. Jia, "Feature Extraction and Classification of Citrus Juice by Using an Enhanced L-KSVD on Data Obtained from Electronic Nose," Sensors, vol. 19, no. 916, pp. 1-17, 2019. 
[28] Y. Wu, T. Liu, S. Ling, J. Szymanski, W. Zhang, S. Su, "Air Quality Monitoring for Vulnerable Groups in Residential Environments Using a Multiple Hazard Gas Detector," Sensors, vol. 19, no. 362, pp. 1-16, 2019.

[29] X. Zhan, X. Guan, R. Wu, Z. Wang, Y. Wang, G. Li, "Discrimination between Alternative Herbal Medicines from Different Categories with the Electronic Nose," Sensors, vol. 18, no. 2936, pp. 1-15, 2018.

[30] C. Deng, K. Lv, D. Shi, B. Yang, S. Yu, Z. He, J. Yan, "Enhancing the Discrimination Ability of a Gas Sensor Array Based on a Novel Feature Selection and Fusion Framework," Sensors, vol. 18, no. 1909, pp. 1-17, 2018.
[31] Juan Guerrero-Ibáñez, S. Zeadally, Juan Contreras-Castillo, "Sensor Technologies for Intelligent Transportation Systems," Sensors, vol. 18, no. 1212, pp. 1-24, 2018.

[32] J. Contreras-Castillo, S. Zeadally, J. Guerrero -Ibáñez, "A seven-layered model architecture for Internet of Vehicles," Journal of Information and Telecommunication, vol. 1, no. 1, pp. 4-22, 2017.

[33] R. Dhall, V. Solanki, "An IoT Based Predictive Connected Car Maintenance Approach," Machines, vol. 4, no. 3, pp. 16-22, 2017.

[34] E. Hamida, H. Noura, W. Znaidi "Security of Cooperative Intelligent Transport Systems: Standards, Threats Analysis and Cryptographic Countermeasures," Electronics, vol. 4, pp. 380-423, 2015. 